\title{
Activation of a Murine Autoreactive B cell by Immunization with Human Recombinant Autoantigen La/SS-B: Characterization of the Autoepitope
}

\author{
Helmut Tröster ${ }^{\star}$, Holger Bartsch ${ }^{\star}$, Rolf Klein ${ }^{\star}$, \\ Thomas E. Metzger ${ }^{\star}$, Gregor Pollak ${ }^{\star}$, Imre Semsei ${ }^{\star}$, \\ Martin Schwemmle†, Ger J. M. Pruijn $\ddagger$, Walther J. van Venrooij \\ and Michael Bachmann* \\ *Institut für Physiologische Chemie, fohannes-Gutenberg Universität, Duesbergweg 6, \\ D-55099 Mainz, Germany; †Institut für Virologie, Albert-Ludwigs-Universität \\ Freiburg, Hermann-Herder Straße 11, D-79104 Freiburg, Germany; $\ddagger$ University \\ of Nijmegen, Department of Biochemistry, PB 9101, NL-6500 HB Nijmegen, \\ The Netherlands
}

(Received 30 March 1995 and accepted 16 August 1995)

\begin{abstract}
Immunization of Balb/c mice with a homogeneously purified recombinant human La/SS-B protein resulted in activation of an autoreactive $B$ cell secreting a novel monoclonal anti-La antibody termed La4B6. La4B6 reacted with La protein from a variety of sources including human, bovine, rat and mouse. ATP blocked the binding of La4B6 to recombinant La protein. The human epitope was identified as consisting of the amino acid sequence SKGRRFKGKGKGN, which includes the proposed ATP-binding site of the La protein. In the human and bovine La protein, the epitope exists as a continuous amino acid sequence. In rat and mouse the epitope was found to consist of the amino acid sequence SKG interrupted by a species-specific insert of 16 amino acids, and followed by the second half of the epitope, the amino acid sequence RRFKGKGKGN. Our data suggest that in the case of the rat and mouse La proteins the two separated parts of the epitope are able to form a conformational epitope which looks similar to the continuous human epitope. $\quad$ (C) 1995 Academic Press Limited
\end{abstract}

Abbreviations: aa, amino acid; cLSM, confocal Laser Scanning Microscopy; ECL, enhanced chemoluminescence; IPTG, isopropyl $\beta$-D-thiogalactopyranoside; NBT, 4-nitro-blue-tetrazolium chloride; BCIP, 5-bromo-4-chloro-3-indolyl phosphate; nt, nucleotide; PVDF, polyvinylidene fluoride; pSS, primary Sjögren's

Address correspondence to: Dr Michael Bachmann, Institut für Physiologische Chemie, JohannesGutenberg Universität, Duesbergweg 6, D-55099 Mainz, Germany. 
syndrome; scRNP, small cytoplasmic ribonucleoprotein; snRNP, small nuclear ribonucleoprotein; SS-B, Sjögren's syndrome associated antigen B (i.e. La).

\section{Introduction}

Sera of patients with systemic immuno-inflammatory autoimmune diseases such as Systemic Lupus Erythematosus or primary Sjögren's syndrome frequently contain autoantibodies to nuclear antigens. One of the targets of these autoantibodies is the $\mathrm{La} / \mathrm{SS}-\mathrm{B}$ antigen, a protein with a molecular weight of about $47-\mathrm{kD}[1,2]$. In the nucleus the La protein may function as a transcription termination factor of RNA polymerase III $[3,4]$. This function is in accord with association of the La protein with nearly all RNA polymerase III transcripts including precursors of $5 S$ RNA, tRNA and 7S RNA, the Y RNAs and some viral RNAs [5]. In agreement with its function as a transcription termination factor the La protein was characterized as an enzyme being able to melt DNA/RNA hybrids and presumably dsRNA [6]. In recent studies Meerovitch et al. characterized a cellular cytoplasmic protein, involved in internal initiation of translation of poliovirus mRNAs, as the La protein [7]. Subsequently it was shown that $\mathrm{La}$ is indeed an ATP-dependent dsRNAunwinding enzyme [8]. In agreement with such unwinding activity, Topfer and coworkers identified a putative ATP-binding motif [9] which is known to be conserved in dsRNA unwinding enzymes [10]. In a recent report Semsei et al. [11] described this proposed ATP-binding site as having been conserved during evolution but containing a species-specific insert in the rat and mouse La protein. The species-specific insert was characterized to be a partial repeat of flanking sequences [11]. According to the mimicry hypothesis, such a region could be a possible target for experimentally inducible autoimmunization.

In this report we describe the induction of a novel monoclonal murine anti-La autoantibody, which was elicited by immunization of mice with a recombinant. human La protein. Epitope mapping studies support the idea that the speciesspecific insert might be involved in the experimentally induced autoimmune reaction.

\section{Materials and methods}

Anti-human antibodies conjugated with Fluorescein (FITC) and anti-mouse antibodies conjugated with Cy3 were purchased from Medac (Hamburg, Germany). Blocking reagent was obtained from Boehringer Mannheim (Mannheim, Germany). Anti-mouse IgG conjugated with peroxidase developed in sheep $\left(\left(\mathrm{Fab}^{\prime}\right)_{2}\right.$ fragments) adsorbed with human serum proteins $(\mathrm{A}-7282 ; 20 \mathrm{U} / \mathrm{ml})$, anti-human IgG ( $\gamma$-chain specific) conjugated with alkaline phosphatase (A3150; $8 \mathrm{U} / \mathrm{mol})$, anti-mouse IgG developed in sheep ((Fab' $)_{2}$ fragments) adsorbed with human serum proteins (A-0532) and isopropyl $\beta$-D-thiogalactopyranoside (IPTG) were obtained from Sigma (St Louis, MA, USA). The enhanced chemoluminescence (ECL) Western blotting detection reagents were from Amersham-Buchler (Braunschweig, Germany). 4-Nitro-blue-tetrazolium chloride (NBT) and 5- 
bromo-4-chloro-3-indolyl phosphate (BCIP) from Roth (Karlsruhe, Germany). Synthetic peptides were purchased from the protein routine laboratory of the Johannes-Gutenberg University of Mainz, Dep. of Physiol. Chem. and Pathobiochem. (Mainz, Germany). PVDF-membrane (IPVH 00010 ; pore size $0.45 \mu \mathrm{m}$ ) was obtained from Millipore (Bedford, MA, USA), and nitrocellulose filters (BA83/21) from Schleicher and Schüll (Dassel, Germany).

\section{Monoclonal antibodies and sera}

Immunization, development and subcloning of the hybridomas as well as ELISA were performed as described previously [12]. The immunization was performed in three steps. In each step $50 \mu \mathrm{g}$ of the gel purified recombinant human La protein was injected intraperitoneally into $\mathrm{Balb} / \mathrm{c}$ AnHan mice.

\section{Monospecific patient anti-La antibody}

Human monospecific anti-La antibody was prepared by adsorption of a monospecific anti-La serum $(\mathrm{Ma} ; 6)$ to the recombinant human $\mathrm{La}$ protein covalently linked to Sepharose-4B. Elution of the bound anti-La antibody was performed as described [13].

\section{Peptide ELISA}

ELISA plates were prepared by coating $200 \mu$ of peptide solution containing $2 \mu \mathrm{g}$ of the respective peptide dissolved in Tris/ $\mathrm{HCl}(\mathrm{pH} \mathrm{7.4}$, containing $150 \mathrm{mM} \mathrm{NaCl}$, $0.1 \%$ Tween 20 (TBST)). The plates were coated overnight at $4^{\circ} \mathrm{C}$, blocked with blocking reagent $(4 \%)$ for $4 \mathrm{~h}$ at $4^{\circ} \mathrm{C}$, and washed with TBST $(3 \times 5 \mathrm{~min})$. Then cell culture supernatant of the hybridoma secreting the anti-La mAb La4B6 was added, incubated for $30 \mathrm{~min}$ and the wells were washed with TBST. Incubation with the secondary anti-mouse antibody conjugated with peroxidase was performed in TBST containing $0.5 \%$ blocking reagent for $30 \mathrm{~min}$. After washing with TBST $(3 \times 5 \mathrm{~min})$ the formed immune complexes were detected using o-Phenylenediamine as substrate. A maximum O.D. of 0.68 was measured for peptide 1 (see Figure 5) and set to $100 \%$.

\section{Cell culture and staining procedures}

The following cell lines were used: Human XPTA cells (13-15), bovine MDBK cells (ATCC CCL 22), rat PC12 cells (ATCC CRL 1721) and mouse 3T3 cells (ATCC CCL 92).

Cells were grown to confluency on coverslips in RPMI 1640 or DMEM medium containing $10 \% \mathrm{FCS}$ and fixed with methanol containing $0.02 \%$ EGTA at $-20^{\circ} \mathrm{C}$ for $1 \mathrm{~h}$. Indirect immunofluorescence of cells with the anti-La mAb was performed by incubating the fixed cells, which had been rehydrated for $5 \mathrm{~min}$ with PBS, with cell culture supernatant for at least $15 \mathrm{~min}$. The cells were washed with PBS (5 $\mathrm{min}$ ) and the bound anti-La mAb was detected using Cy3-conjugated goat anti-mouse antibody. The staining of rat and mouse cells proved to be critical. Best 
results were obtained when the staining procedure was performed on ice using protein A purified anti-La mAb dialyzed against PBS. Indirect immunofluorescence labelling using immunoadsorbed patient anti-La antibody was performed using the same procedure. The bound human anti-La antibody was detected with speciesspecific anti-human antibody conjugated with FITC. Four double immunofluorescence, the cells which had already been stained with the anti-La mAb La4B6, as described above, were subsequently incubated with the patient anti-La antibody. In all cases the incubation time for the secondary antibody was $15 \mathrm{~min}$. The staining occurred at room temperature and the unbound secondary antibodies were removed by washing with PBS (twice, $5 \mathrm{~min}$ ). The stained cells were mounted using PBS/glycerol (1:1 [v/v]).

\section{Epifluorescence microscopy}

Stained cells were analysed with a Zeiss Axiophot microscope equipped with epifluorescence optics and filter systems specific for the fluorescence dyes used [12-15].

\section{Confocal laser scanning microscopy}

Confocal laser scanning microscopy (cLSM) was performed using a Zeiss LSM 10. Cells were cut automatically into horizontal sections $(512 \times 512$ pixels $/ 8$ bit, objective lenses Plan-Neofluar $40 \mathrm{x} / 1.3$ oil). Evaluation of the stored stacks of the horizontal optical sections was performed with the LSM 10 image processing unit.

\section{Preparation of cell extracts}

Monolayer cells, grown to confluency, were lysed in hot SDS-PAGE sample solution $\left(0.1 \mathrm{M} \mathrm{Na}_{2} \mathrm{HPO}_{4}\right.$, pH $8.3 ; 20 \%$ [w/v] sucrose, $1 \%$ SDS, $1 \mathrm{mM}$ PMSF; $500 \mu \mathrm{l}$ per $200 \mathrm{ml}$ flask). The protein extract was heated in an Eppendorf tube for $5 \mathrm{~min}$ at $95^{\circ} \mathrm{C}$, centrifuged for $15 \mathrm{~min}$ at $11000 \times \mathrm{g}$ and frozen until use.

\section{SDS polyacrylamide gel electrophoresis and immunoblotting}

SDS-PAGE was performed according to Laemmli [16] and immunoblotting to PVDF-membrane according to Matsudaira [17]. Prior to electrophoresis the samples were heated for $3 \mathrm{~min}$ to $95^{\circ} \mathrm{C}$ in the presence of $1 \mathrm{mM} \beta$-mercaptoethanol if not stated otherwise. Immune complexes on the blots were visualized using either the ECL-Western blotting detection system or the alkaline phosphatase system using NBT-X-phosphate or BCIP/NBT as substrates.

\section{Isolation of La cDNAs and preparation of deletion mutants}

A human cDNA library was prepared from peripheral blood lymphocytes of a patient with pSS [11] and screened using patient anti-La serum Ma [6]. The two longest clones (termed La19 and La23) were sequenced (EMBL Acc. No.:

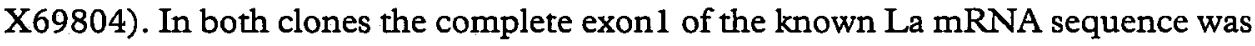


replaced by an alternative 5 -terminus. This difference is not relevant for the present study as the identified epitope (see Results) is located in the C-terminus of the La protein. Deletion mutants were made from La19 and La23, in both cases using the same restriction site. However, as the La insert in La19 is inverted, expression of deletion mutants obtained from this clone (Lal9) resulted in La proteins shortened from the C-terminus, while expression of the deletion mutants obtained from the clone $\mathrm{La} 23$ resulted in La proteins with $\mathrm{N}$-terminal truncations.

Due to the inversion of the $\mathrm{La}$ insert in clone Lal9, the expression of the C-terminally truncated La proteins did not depend on (1) the $\beta$ - $G$ al derived ATG and (2) the reading frame. Similarly, all the deletion mutants derived from La23 expressed immunoreactive La protein independent on the reading frame of the $\beta$-Gal derived ATG until the internal ATG (encoding aa $M$ (223)) was deleted. Deletion mutants lacking the aa $M$ (223) expressed immunoreactive La protein only when the $\beta$-Gal derived ATG was in the reading frame of the La protein, which indicates that an internal initiation may occur at this ATG in E. coli. Deletion mutants (for example, clones 23 to 25 ; Figure $3 \mathrm{~A}$ ) not containing the La insert in the reading frame of the $\beta$-Gal derived ATG were not or less efficiently expressed and therefore not useful.

DNA for preparation of the deletion mutants was prepared from small scale cultures (up to $12 \mathrm{ml}$ ) using the protocol of Holmes and Quigley [18] modified according to Sambrook et al. [19]. To create deletion clones the method originally described by Henikoff [20] was used. The DNA to be deleted was doubly digested with either Sac I (La23) or BstX I (La19) and EcoRI from which site deletions started. EcoRI represents the $5^{\prime}$-cloning site and served as the initiation site of deletion. DNA of each deletion mutant was prepared and sequenced as described [11].

\section{Expression of recombinant La protein}

The human recombinant $\mathrm{La}$ protein was expressed in $E$. coli strain BL21(DE3)pLysS as described by Slobbe et al. [21]. The La protein was subsequently purified from the bacterial lysate by ion exchange chromatography using DEAE cellulose (Whatman DE52) and phosphocellulose (Whatman P11) resins. DEAE cellulose column chromatography was performed with a buffer containing $25 \mathrm{mM}$ Tris/ $\mathrm{HCl}, \mathrm{pH} 8.0,100 \mathrm{mM} \mathrm{NaCl}, 1 \mathrm{mM}$ EDTA, $1 \mathrm{mM}$ dithioerythritol. The flow through of the DEAE column, containing the La protein, was applied to the phosphocellulose column, which was equilibrated with $50 \mathrm{mM}$ HEPES-KOH, $\mathrm{pH} 7.9,100 \mathrm{mM} \mathrm{NaCl}, 1 \mathrm{mM}$ dithioerythritol. Bound material was eluted with a linear salt gradient and the $\mathrm{La}$ protein eluted at approximately $700 \mathrm{mM} \mathrm{NaCl}$. Alternatively, expression of $\mathrm{La}$ inserts in pBluescript II SK(-) were performed after transformation into the bacterial strain BL21(DE3)pLysS using the Studier system [22] modified according to a protein expression protocol of Stratagene (Heidelberg, Germany). After expression the bacterial suspensions were centrifuged for $15 \mathrm{~min}$ at $1600 \times g\left(4^{\circ} \mathrm{C}\right)$ and the pelleted bacteria were resuspended in lysis buffer (50 mM Tris/HCl, pH 8.0; $10 \mathrm{mM}$ EDTA, $1 \mathrm{mM} \mathrm{PMSF,} 10 \%$ [w/v] sucrose) on ice. Lysozyme was added to a final concentration of $1 \mathrm{mg} / \mathrm{ml}$. After 10 min Triton X-100 was added to a final concentration of $0.1 \%[v / v]$. After a further 
$10 \mathrm{~min}$ the samples were centrifuged in an Eppendorf centrifuge at $11000 \times g$ for $1 \mathrm{~h}$. The supernatants were used as crude bacterial extracts.

\section{Colony screening}

The length of the inserts of the deletion mutants had been characterized by restriction enzyme fragment length analysis. The different clones were arranged according to the length of the $\mathrm{La}$ inserts. One $\mu \mathrm{l}$ of a suspension of the respective clone was pipetted from a glycerol stock $(17 \%[\mathrm{v} / \mathrm{v}])$ directly onto a sterile nitrocellulose filter placed upon an ampicillin agar plate $(150 \mu \mathrm{g} / \mathrm{ml})$. The filter plate was incubated overnight at $37^{\circ} \mathrm{C}$ and further processed according to Sambrook et al. [19]. Immune reactions on the filter using the anti-La mAb La4B6 were performed as described for the immunoblotting protocol using the alkaline phosphatase BCIP/NBT method.

\section{Results \\ Development of anti-La mAb La4B6}

The human recombinant La protein was expressed in E. coli and isolated from total bacterial extracts by two ion exchange chromatographic steps followed by immunoaffinity chromatography (see Materials and Methods). Although the isolated recombinant La protein appeared to be homogeneously purified after these steps, the La protein fraction used for immunization was further purified on a preparative denaturing SDS-polyacrylamide gel. The band corresponding to the La protein was excised and the La protein was eluted from the gel. This isolated La protein fraction was essentially free of any contaminating bacterial protein and of any degradation product of the La protein. After SDS-PAGE and transfer to PVDF-membrane, this La protein fraction gave a single band when stained with Coomassie-brilliant blue (Figure 1A, lane a). As expected, this protein band reacted strongly with the patient anti-La antibody (Figure 1A, lane b). After immunization and fusion the resulting hybridoma lines were screened by ELISA using the immunoaffinity purified recombinant La protein (see Materials and Methods).

Only one of two obtained IgG class mAbs, termed La4B6, and none of the 9 IgM $m A b s$ reacted with the $\mathrm{La}$ protein after SDS-PAGE and immunoblotting (Figure $1 \mathrm{~B}$, lanes $\mathrm{c}$ to $\mathrm{f}$ ). Interestingly, La4B6 reacted with the La protein of total extracts obtained from a variety of cell lines including human (Figure $1 \mathrm{~B}$, lane $\mathrm{c}$ ), mouse (Figure 1B, lane d), rat (Figure 1B, lane e) and bovine (Figure 1B, lane $f$ ). No cross-reaction with another cellular protein could be detected, even when using the sensitive ECL system for detection of immune complexes.

Due to the presence of protease-sensitive PEST-regions [2], an affinity-purified recombinant human $\mathrm{La}$ protein preparation mostly contains the well-known $\mathrm{N}$-terminal $29 \mathrm{kDa}$ and the C-terminal $24 \mathrm{kDa}$ domains of the La protein in addition to the full-length $\mathrm{La}$ protein. Both $\mathrm{La}$ fragments react with patient anti-La antibody (Figure 1C, lane g), although with this particular patient serum (Ma) [6] the reactivity to the $\mathrm{C}$-terminus was less intensive (Figure $1 \mathrm{C}$, lane $\mathrm{h}$ which depicts a shorter exposure time of lane $\mathrm{g}$ ). When the same blot was incubated with La4B6, 


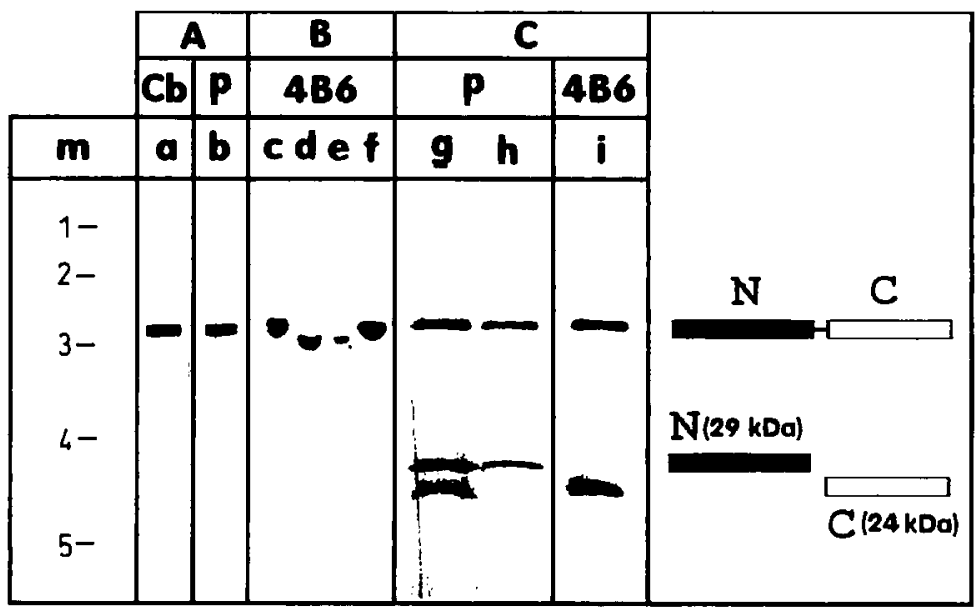

Figure 1. Characterization of the recombinant $\mathrm{La}$ protein and anti-La antibodies. (A) Purified full-length recombinant human La protein fraction stained with Coomassie-Brilliant Blue (lane $a, C b$ ), and, after transfer to nitrocellulose, by the patient (Ma) anti-La antibody (lane b, P). (B) Immunoblotting patterns of $\mathrm{mAb}$ La4B6. The anti-La mAb La4B6 was allowed to react with the La protein on blots containing total extracts of human (lane c), mouse (lane d), rat (lane e) and bovine (lane f) cells. (C) Purified bacterially expressed recombinant La protein, containing $\mathrm{N}$ - and $\mathrm{C}$-terminal degradation products in addition to the full length product, was blotted and incubated with patient anti-La antibody (lane $\mathrm{g}$ ) and mAb La4B6 (lane i). lane $\mathrm{h}$ : A shorter exposure of lane $\mathrm{g}$.

the $\mathrm{mAb}$ reacted strongly with the $\mathrm{C}$-terminal but not with the $\mathrm{N}$-terminal fragment (Figure 1C, lane i). From these results we conclude that (1) the anti-La mAb La4B6 specifically reacts with the La protein and (2) the epitope locates within the C-terminal fragment of the La protein.

\section{Characterization of the anti-La mAb La4B6 by epifluorescence microscopy}

Epifluorescence microscopy revealed that the anti-La mAb gives a predominantly nuclear staining pattern on cells derived from human (Figure 2a) and mouse (Figure $2 \mathrm{~d}$ ) as well as from bovine and rat (data not shown) cell lines. In double labelling experiments, the staining pattern of the anti-La mAb appeared to be similar, but not identical, to the pattern obtained with purified patient anti-La antibody (compare Figure 2, lanes a and d with lanes $c$ and $f$ ).

\section{Characterization of the anti-La $m A$ b La4B6 by confocal laser scanning microscopy}

In a recent report it was shown that the use of cLSM combined with image processing allows the identification of nuclear substructures, which are usually not directly visible by classical epifluorescence microscopy [23]. Using this technique, which allows fading out of the homogeneous nuclear staining that is usually obtained after epifluorescence microscopy (Figure 2a, c, d, f, h), mAb La4B6 produced a punctuated nuclear staining pattern (Figure 2i), while the affinitypurified human anti-La antibody gave a more coarse nuclear staining pattern (Figure 2j). To obtain the images shown in Figures $2 i$ and $j$ the entire cell was cut 

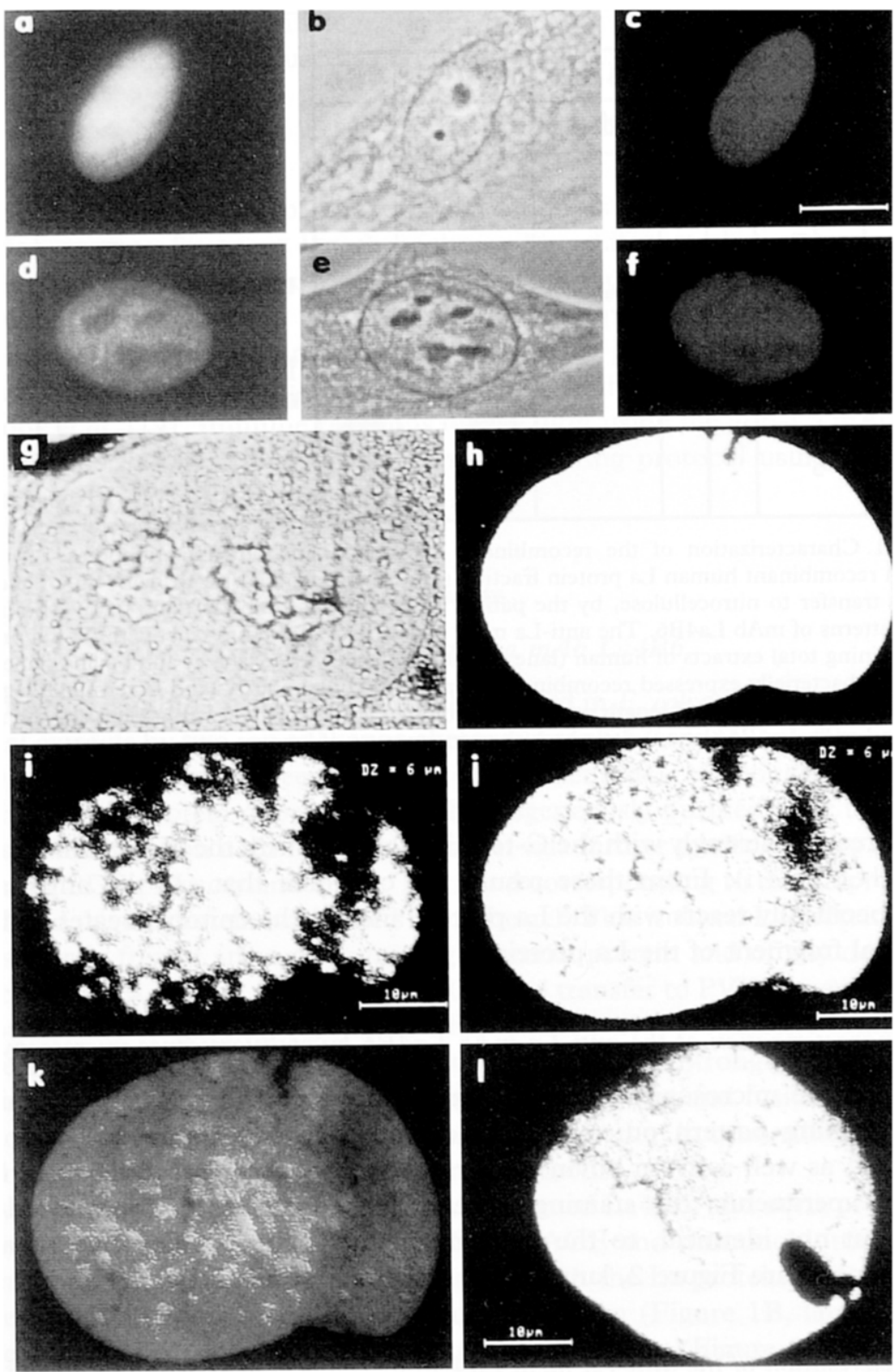

Figure 2. Images of mAb La4B6 and patient ( $\mathrm{Ma}$ ) anti-La antibody obtained with epifluorescence and confocal laser scanning microscopy. Human (XPTA; a to $c$ ), and mouse ( $3 \mathrm{~T} 3$; d to f) cells were stained with either the anti-La mAb La4B6 ( $\mathrm{a}, \mathrm{d}$ ) or patient anti-La antibody (c, f) purified by immunoadsorption on recombinant human La protein. The corresponding phase contrast images are shown in lanes (b) and (e). Bar in (c) $=4 \mu \mathrm{m}$. The staining pattern of XPTA cells was analysed in either the non-confocal mode to obtain an epifluorescence pattern $(h)$ or in the confocal mode $(i, j)$. The images $(i, j)$ were prepared by overlaying a stored stack of optical sections obtained by z-sectioning of the cell with the cLSM into 13 different planes at a distance of $0.5 \mu \mathrm{m}$. The image (k) shows a coloured overlay of (i) and (j). The corresponding phase contrast image is shown in (g). A similar staining partern as shown in (j) for the patient anti-La antibody was also obtained when the cells were not pre-stained with the mAb but stained with the patient anti-La antibody only (1). 
in z-dimension with the cLSM, resulting in 13 sections for either the FITC-stained patient anti-La antibody or the Cy3 stained anti-La mAb La4B6. Then an overlay of the stored stacks of optical sections was prepared for the series of optical sections of either the anti-La mAb La4B6 (Figure 2i) or the patient's anti-La antibodies (Figure $2 \mathrm{j}$ ). Finally a coloured overlay of the resulting two images was made to more clearly show the differences between the two staining patterns (Figure $2 \mathrm{k}$ ). From these results we conclude that, as might be expected, neither the anti-La mAb La4B6 nor the patient anti-La antibody used was able to stain all La protein molecules in a cell during immunofluorescence.

\section{Characterization of the antigenic determinant recognized by the anti-La mAb La4B6}

In order to characterize the $\mathbf{B}$ cell epitope and to obtain an explanation for the different staining patterns of the patient anti-La antibody and the anti-La mAb La4B6, we decided to perform epitope mapping experiments. In a first step deletion mutants were prepared in which the La protein was truncated from either the $\mathrm{N}$ - or the C-terminus. In the next step the epitope region was further bordered by the use of synthetic peptides.

\section{Epitope analysis using deletion mutants of the recombinant human La protein}

The deletion mutants used for the epitope mapping experiments were prepared from the human La cDNA clones La19 and La23 (see Materials and Methods). Both clones contain the complete coding region of the La protein. However, in the case of $\mathrm{La} 19$ the $\mathrm{La}$ insert was inverted during cloning in the pBluescript vector. In consequence, deletion of these two clones from the same site resulted in deletion mutants expressing the La proteins being truncated from either the $\mathrm{N}$-terminus (derived from La23) or the C-terminus (derived from La19).

In total, 85 deletion mutants were selected. Clones 1 to 38 were derived from La23 and clones 39 to 85 from La 19. The clones were analysed by colony screening (Figure 3A). On the filter the clones were arranged according to the length of their inserts. Neighbouring clones differed about 30 to $100 \mathrm{nts}$ in length. As explained in Materials and Methods, clones 23-25 were not or less efficiently expressed and therefore not very useful. Clone 22 contained the smallest $\mathrm{La}$ insert being still reactive with the anti- $\mathrm{La} \mathrm{mAb}$ after expression into a $\mathrm{N}$-terminally truncated La protein. It starts with the aa $\mathrm{V}$ (305). The longest non-reactive clone expressing La protein truncated from the $\mathrm{N}$-terminus is clone 26 , which starts with aa $\mathrm{A}(384)$. In consequence, the $\mathrm{N}$-terminal deletion mutants locate the epitope between aa 305 and aa 384 .

In the case of clones expressing the La protein truncated from the C-terminus the most interesting clones were clone 54 (ending at aa G (342)) which was still reactive, and clone 55 (ending at aa $S(331)$ ) which was the longest non-reactive clone. As summarized in Figure 3B, the colony screening experiments indicated that the epitope was located between aa 331 and 342 .

The results of the colony screening experiments were confirmed by analysis of the bacterial extracts by immunoblotting (Figure $4 \mathrm{~A}-\mathrm{C}$ ). In this type of experiment extracts obtained from bacteria expressing either the full length clone La19 (Figure 


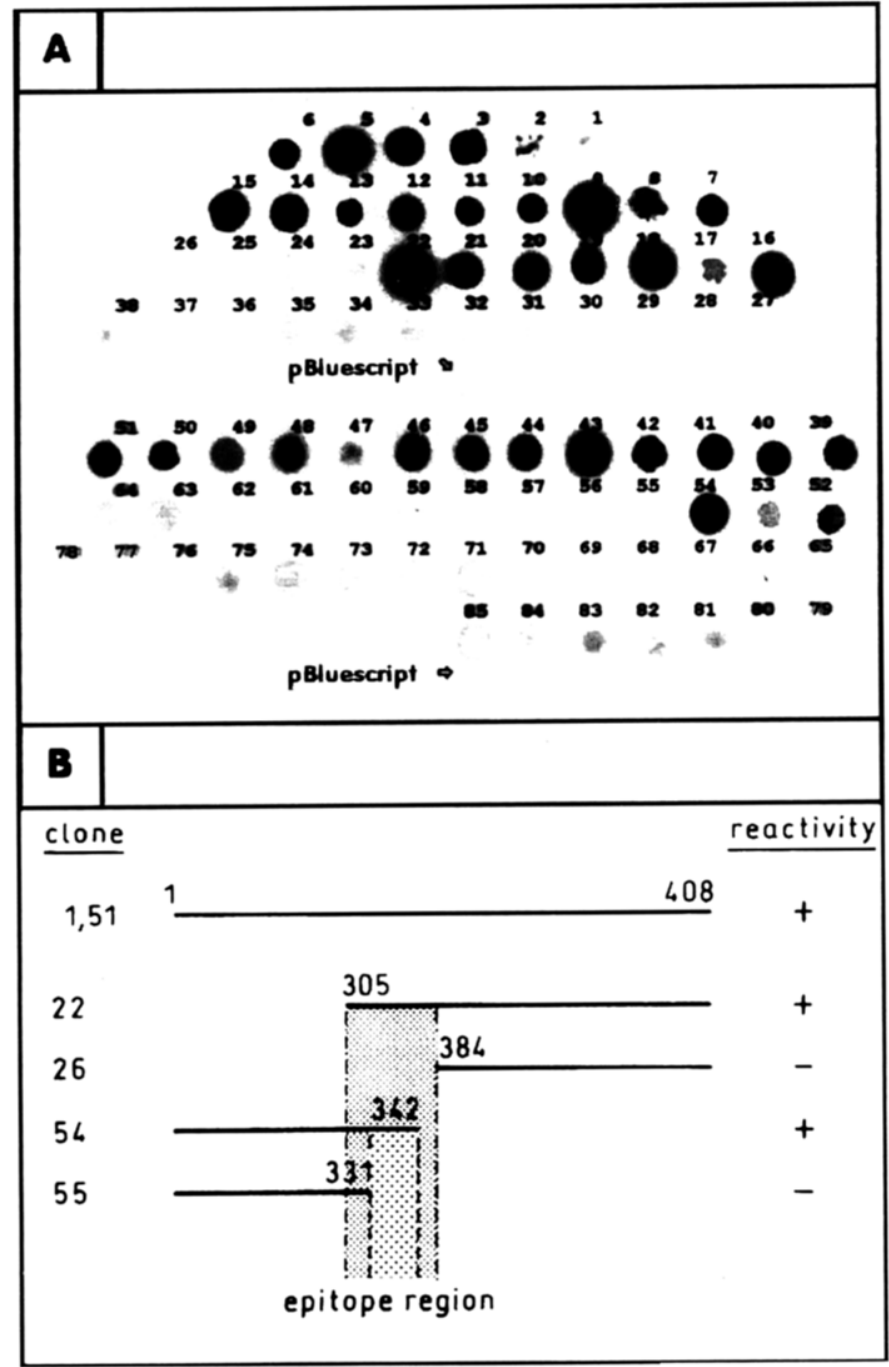

Figure 3. La4B6 epitope mapping by deletion mutant analysis. (A) Bacteria expressing deletion mutants of the La protein truncated from either the N-terminus ( 1 to 38 ) or the C-terminus (39 to 85) were analysed for reactivity with the anti-La mAb La4B6. Clone 54 encodes a La protein mutant ending at aa $G$ (342), while clone 55 ends with the aa S (331). The clones 22 and 26 started with the aa V (305) or aa A (384), respectively. Clones 23 to 25 were not able to express La protein derivatives (see Materials and Methods). The non-reactive clones ( 23 to 38 and 55 to 85 ) gave similar background staining intensities as the bacteria containing the pBluescript vector only (arrow). (B) Summary of the results of the colony screening experiment. The $\mathrm{N}$-terminal deletion mutants 22 and 26 locate the epitope between aa 305 and 384; the C-terminal deletion mutants 54 and 55 locate it between aa 331 and 342 . The reactivity of the various clones is listed on the right.

$4 \mathrm{~A}-\mathrm{C}$, lanes $\mathrm{b}$ and $\mathrm{c}$ ) or the deletion mutants clone 55 (Figure $4 \mathrm{~A}-\mathrm{C}$, lanes e and f) or clone 54 (Figure $4 \mathrm{~A}-\mathrm{C}$, lanes $\mathrm{g}$ and $\mathrm{h}$ ) were analysed by immunoblotting. Total extracts of bacteria transformed with the pBluescript vector lacking an insert were used as a negative control (Figure 4A-C, lanes a and d). In the first part of the experiment the transferred proteins were probed with the anti-La mAb La4B6. The 


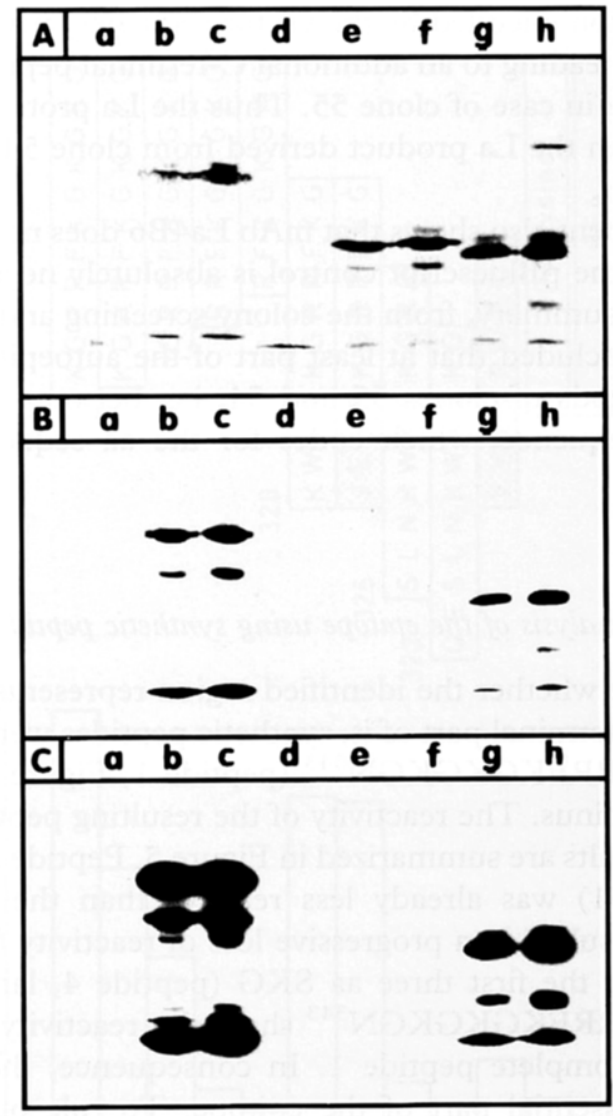

Figure 4. Analysis of reactivity of patient antibody and mAb La4B6 with delecion mutants by immunoblotting. (A) Extracts from bacteria not expressing any La product (lanes a, d) or expressing either full length La (lanes $b, c$ ) or deletion mutants 55 (lanes $e, f$ ) or 54 (lanes $g$, $h$ ) were allowed to react with patient anti-La antibody in order to ensure that comparable amounts of La product were present on the blots. (B) The same blots as in (A) were incubated with mAb La4B6. (C) Overexposure of the blots shown in (B).

immune complexes formed were visualized using the ECL system (Figure $4 \mathrm{~B}$ and C). After detection, the immune complexes were eluted from the blot and the same blot was re-incubated with the patient anti-La antibody. As is evident from the blot incubated with the patient anti-La antibody (Figure 4A), the bacterial extract of every La clone contained the respective immunoreactive La protein. In contrast to the pattern obtained with the patient anti-La antibody but in agreement with the colony screening experiments, the anti-La mAb La4B6 did react with deletion mutant 54 (Figure 4B and $C$, lanes $g$ and $h$ ) but not with deletion mutant 55 (Figure $4 \mathrm{~B}$ and $\mathrm{C}$, lanes $\mathrm{e}$ and $\mathrm{f}$ ).

As can be seen in Figure 4A, the La protein derived from clone 54, presumed to be 11 aa longer than the La protein derived from clone 55, migrates faster during SDS-PAGE. This is explained as follows. During preparation of the deletion mutants, clones 54 and 55 lost the C-terminal stop codon of the La cDNA. As a consequence, termination of translation of the respective La clone takes place at the 
first in-frame stopcodon encoded by the vector sequence, which differs in position between both clones, leading to an additional C-terminal peptide of 11 aa in case of clone 54 and of 47 aa in case of clone 55 . Thus the La protein derived from clone 55 is 25 aa longer than the La product derived from clone 54 explaining the faster migration of the latter.

Finally this experiment also shows that $\mathrm{mAb}$ La4B6 does not cross-react with any bacterial epitope, as the pBluescript control is absolutely negative (Figure $4 \mathrm{~B}$ and $C$, lanes a and $d$ ). In summary, from the colony screening and the immunoblotting analyses it can be concluded that at least part of the autoepitope is located in the region between the ends of clones 55 and 54, i.e. between nts 992-1024 of the human La cDNA sequence, which codes for the aa sequence ${ }^{332}$ KGRRFKG$\mathrm{KGKG}^{342}$.

\section{Analysis of the epitope using synthetic peptides}

In order to determine whether the identified region represents either the complete epitope or a $\mathrm{N}$ - or C-terminal part of it, synthetic peptides were made based on the aa sequence ${ }^{331}$ SKGRRFKGKGKGN ${ }^{343}$ (peptide 1, Figure 5) which was shortened from the $\mathrm{N}$-terminus. The reactivity of the resulting peptides was determined by ELISA and the results are summarized in Figure 5. Peptide 2 (lane 2) lacking the $\mathrm{N}$-terminal aa $\mathrm{S}$ (331) was already less reactive than the complete sequence. Further shortening resulted in a progressive loss of reactivity (Figure 5, peptides 3 to 5). After removing the first three aa SKG (peptide 4, lane 4), the remaining peptide sequence ${ }^{334} \mathrm{RRFKGKGKGN}{ }^{343}$ showed a reactivity of less than $15 \%$ as compared with the complete peptide 1 . In consequence, the aa sequence SKG appeared to be an essential part of the epitope. To rule out that the observed decrease in reactivity was due to a gradual loss of binding affinity of the respective peptide to the ELISA plate, additional peptides with an identical length of 11 aa were made. Each of these dodecamers contained (1) a part of the epitope including the aa, either $S_{K G}{ }^{33}$ (peptide 9, Figure 5), or $S K G R R^{335}$ (peptide 8) or SKGRRFKG ${ }^{338}$ (peptide 6) and (2) the respective upstream flanking sequence. Only peptide $6\left({ }^{328}\right.$ KWKSKGRRFKG $\left.{ }^{338}\right)$ was well recognized by mAb La4B6. Replacing the three N-terminal aa KWK of this peptide with the unrelated sequence JEV, as in peptide 7 , did not affect the reactivity. From these results we concluded that the epitope probably starts at position 331 (S).

As shown in Figure 5, peptide 6 was somewhat less reactive than peptide 1 . This could be explained by the different length of the peptides (13 aa (peptide 1) vs 11 aa (peptide 6)) or by the five additional C-terminal aa KGKGN in peptide 1 which might be involved in formation of the complete epitope. To differentiate between these two possibilities the two peptides were covalently linked to BSA at the same molar ratio. When these conjugates were analysed in comparison to BSA the same difference in activity as was found earlier for the unconjugated peptides was obtained (data not shown). Therefore, the sequence ${ }^{339} \mathrm{KGKGN}^{343}$ downstream of the sequence ${ }^{331}$ SKGRRFKG ${ }^{338}$ partially contributes to the complete epitope. In parallel the reactivity of recombinant human La protein was measured. BSA conjugated with peptide 1 had $37 \%$ of the reactivity of the recombinant human La 


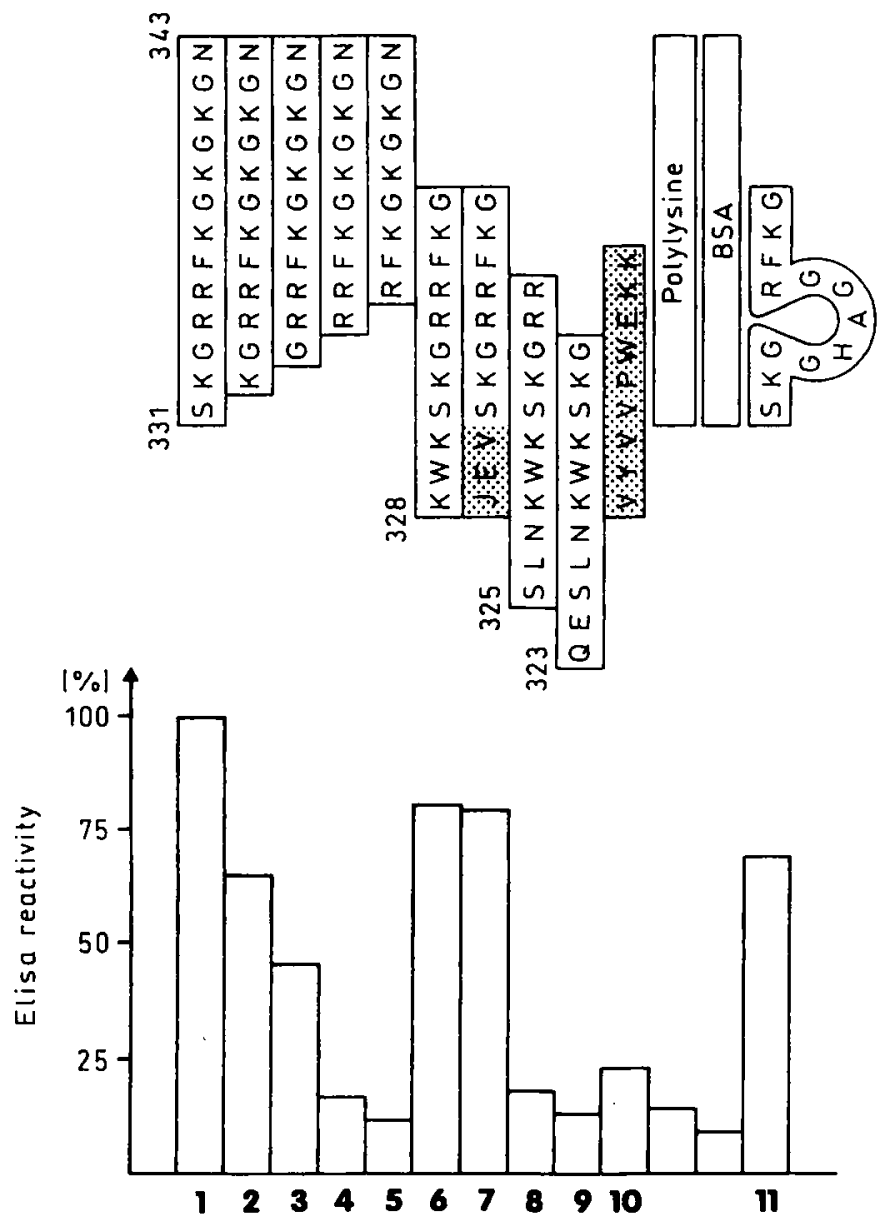

Figure 5. La4B6 epitope mapping using synthetic peptides, each comprising part of the aa sequence of the human epitope region identified by colony screening, ${ }^{331}(\mathrm{~S}) \mathrm{KGRRFKGKGKG(N)}{ }^{343}$, were made. The reactivity of the anti-La mAb to these peptides was measured by ELISA (see Materials and Methods). Background activity levels were measured using polylysine, BSA and a non-related control peptide VYVVPWEKK (lanes 10). lane 1: peptide 1 (aa 331-343). The reactivity of this peptide was set at $100 \%$; lane 2: peptide 2 (aa 332-343); lane 3: peptide 3 (aa 333-343); lane 4: peptide 4 (aa 334-343); lane 5: peptide 5 (aa 335-343); lane 6: peptide 6 (aa 328-338); lane 7: peptide 7, the aa ${ }^{328} \mathrm{KWK}^{330}$ in peptide 6 were changed into JEV; lane 8: peptide 8 (aa 325-335); lane 9: peptide 9 (aa 323-333); lane 11: peptide 11 , consisting of the two epitope parts SKG and RFKG, interrupted by part of the mouse-specific insert.

protein, suggesting that additional sequences in the La protein might be involved in correct epitope formation.

It was noted that the epitope does not exist as a continuous aa sequence in the rat and mouse La proteins. In the mouse $\mathrm{La}$ protein (the aa positions for the mouse $\mathrm{La}$ protein are given in italics) it is split by the species-specific insert ${ }^{333} \mathrm{GHAG}$ GRFKGSHVFTAA ${ }^{348}$ into two parts consisting of the N-terminal aa ${ }^{330} \mathrm{SKG}^{332}$ and the C-terminal aa ${ }^{349}$ RRFKGKGKGN ${ }^{358}$. This C-terminal part RRFKGKGKGN cannot account for the binding of La4B6 to mouse or rat La proteins 


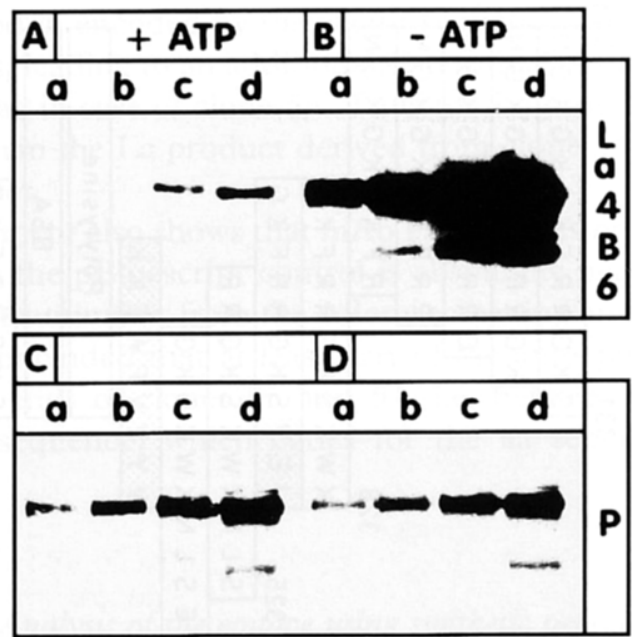

Figure 6. Effect of ATP on binding of the anti-La mAb La4B6 to recombinant human La protein. Increasing amounts of recombinant human La protein ( $A$ to $D$, lanes $\mathrm{a}: 0.2 \mu \mathrm{g}$, lanes b: $0.4 \mu \mathrm{g}$, lanes c: $0.8 \mu \mathrm{g}$, lanes $\mathrm{d}: 1.6 \mu \mathrm{g}$ ) were blotted and incubated with the anti-La mAb La4B6 in the presence of $150 \mathrm{mM}$ of ATP (A) or in the absence of ATP (B). The same blots, after removing La4B6 immuno-complexes by washing with $0.2 \mathrm{M}$ glycine/ $\mathrm{HCl}(\mathrm{pH} 2.3 ; 500 \mathrm{mM} \mathrm{NaCl}$ ), were re-incubated with patient anti-La antibody again in the presence and absence of ATP (C and D).

observed in immunoblotting and immunofluorescence (Figures 1 and 2) since this sequence has only a very low affinity towards the monoclonal antibody (Figure 5, lane 4). Therefore, another synthetic peptide was made containing at the $\mathrm{N}$-terminal side the aa sequence SKG followed by part of the mouse specific insert ${ }^{333}$ GHAGGRFKG ${ }^{341}$, which also contains the partial repeat (RFKG) of the C-terminal epitope sequence. Interestingly, this peptide 11 showed about $80 \%$ of the reactivity exhibited by the corresponding human linear sequence (compare lanes 11 and 1). Consequently (1) the C-terminal part ${ }^{339} \mathrm{KGKGN}^{343}$ of the human epitope ${ }^{331}$ SKGRRFKGKGKGN ${ }^{343}$ is not absolutely required for binding of the anti-La mAb La4B6 and (2) the two parts ${ }^{331} \mathrm{SKG}^{333}$ and ${ }^{334}{ }^{\mathrm{RRFKG}}{ }^{338}$ of the human epitope do not necessarily have to exist as a linear peptide. As the second half of the mouse epitope ${ }^{349} \mathrm{RRFKG} \mathrm{G}^{353}$ is partially $\left({ }^{338} \mathrm{RFKG} \mathrm{G}^{341}\right)$ repeated in the rat and mouse insert it is difficult to decide whether the first or the second or both RFKG sequences are involved in autoepitope formation in the rat and mouse La proteins. In conclusion, our data suggest that the autoepitope in the mouse La protein which is recognized by the mouse mAb La4B6 is a conformational epitope formed by the aa sequence ${ }^{330}$ SKGGHAGGRFKGSHVFTAA RRFKGKGKGN $^{358}$.

\section{Blocking of the epitope by ATP}

Interestingly, the identified epitope region coincides with the predicted ATPbinding site of the La protein. Therefore we analysed the effects of ATP on the reactivity of anti-La mAb La4B6 with recombinant human La protein. For that purpose increasing amounts of recombinant human La protein (Figure 6) were 
blotted and incubated with the mAb La4B6 in the presence of $150 \mathrm{mM}$ ATP (Figure 6A) or in the absence of ATP (Figure 6B). The results clearly indicate that ATP is able to abolish the binding of the mAb La4B6 to the recombinant human La protein. The same blots, after removal of the La4B6 immuno-complexes, were re-incubated with patient anti-La antibody to show that ATP did not detectably influence the recognition of La by patient antibodies. Also, lower concentrations of ATP (10-100 nM) were able to interfere with the binding of mAb La4B6 to La (data not shown). From these experiments we conclude that ATP is able to compete with mAb La4B6 for binding to $\mathrm{La}$, probably by binding to the epitope region recognized by this monoclonal antibody.

\section{Discussion}

Sera of patients with systemic autoimmune diseases frequently contain autoantibodies to the nuclear autoantigen La/SS-B. Mouse monoclonal antibodies directed to the La protein have been described by several groups [6, 12, 25-27] but in most cases these anti-La mAbs either do not cross-react with the mouse La protein [25, 27] or behave differently with respect to their staining pattern and RNA coprecipitation properties if compared to patient anti-La antibodies $[6,12]$. Recently, La cDNA sequences encoding the rat and mouse La protein became available and sequence comparison revealed that species-specific inserts appeared to be present in the human, bovine, rat and mouse La sequences $[9,11]$. To test whether these regions could have autoantigenic properties, mice were immunized with a recombinant human La protein preparation and the resulting hybridomas were analysed for cross-reactivity with the mouse La protein. The anti-La mAb La4B6 was found to react with the human, bovine, rat and mouse La protein both in immunofluorescence and immunoblotting. In epifluorescence microscopical studies the anti-La mAb La4B6 gave a comparable staining pattern to the patient anti-La antibody. However, if the immuno-stainings were analysed with the cLSM technique, differences between the staining patterns of the patient anti-La antibody and the anti-La mAb La4B6 became evident. The discrepancies between the staining patterns were not due to competition of the antibodies during the staining process because they could be observed in double as well as in single immunofluorescence studies (Figure 2).

To explain these discrepancies in staining patterns and to obtain some idea of the mechanism leading to activation of a mouse autoreactive $B$ cell, we characterized the human epitope region and compared it with the sequence of the corresponding mouse La autoepitope. All epitope mapping experiments, including $\mathrm{N}$ - and $\mathrm{C}$-terminal deletion mutants (Figures $1 \mathrm{C}, 3,4$ ), and the use of synthetic peptides (Figure 5) locate the epitope in the C-terminal part of the La protein. In contrast, the patient anti-La antibody used in this study preferentially reacted with the $\mathrm{N}$-terminal part of the La protein (Figure 1C). A possible explanation for the different staining pattern of the mAb La4B6, as compared to the patient antibody, is that the La4B6 epitope on the La protein might not always be accessible.

The epitope sequence could be analysed in detail by the use of synthetic peptides (Figure 5). Taken together, five of the peptides used to characterize the epitope 
region (peptides 3, 6 to 9) had the same length of 11 aa. The number of charged aa within these peptides ranged from 3 (peptides 10,11) to 6 (peptides 1, 2). However, these differences in the number of charged aa seemed not to be relevant. For example, the peptides 6 and 7 showed the same high reactivity although they contain 6 and 4 positively charged aa, respectively, while peptides 8 and 9 containing 5 and 3 positively charged aa, respectively, gave comparable low reactivities.

Recently Topfer et al. [9] identified an ATP-binding motif in the La protein which appeared to be part of the epitope region identified for the anti-La mAb La4B6. In agreement with this suggestion it was found that ATP effectively competes for the binding of mAb La4B6 to the La protein (Figure 6).

Interestingly, the identified human epitope sequence is split in the mouse and rat La protein into two parts by one of the recently identified species specific inserts. As synthetic peptides containing either the N-terminal part of the human epitope (SKG) or the C-terminal part (RRFKGKGKGN) did not react with the anti-La mAb La4B6, while a synthetic peptide containing the two epitope parts separated by the mouse specific insert was well recognized by La4B6, we hypothesize that the three-dimensional organization of the human and mouse La4B6 epitope must be similar. However, in the case of the human La protein the epitope region is formed by a continuous aa sequence, while in the mouse La protein the insert and the two parts of the epitope are probably folded in such a way that a similar arrangement of amino acids crucial for the epitope is obtained. This conformational arrangement might be evolutionarily conserved due to the fact that it represents a functional entity of the La protein (ATP binding site). The difference in folding of this region between the human, mouse and rat La protein might also explain the increased sensitivity to denaturation of the mouse and rat La epitope which was observed in some experiments (data not shown).

Epitope mapping studies have also been performed in previous studies (reviewed in 28). In most cases immunization of mice with human or bovine La protein resulted in species restricted reactivities of the anti-La mAbs $[25,27]$. Two groups immunized mice with bovine La protein and obtained either bovine specific anti-La mAbs [27] or anti-La mAbs cross-reacting with human and bovine but not with mouse La protein [25]. The epitopes recognized by these antibodies are therefore probably directed to less conserved regions and most probably not to functional entities of the La protein [29].

Human autoantibody populations frequently contain antibodies directed to the same epitopic region shown to be targeted by La4B6. Rauh and coworkers described that one of at least three human B-cell epitopes locates within a fragment spanning aa 292-345 [30]. Similarly, Sturgess et al. [31] reported an anti-La reactivity directed to a fragment between aa 305 and 408. Also Chambers et al. [32] and St Clair et al. [33] identified an antigenic region between the aa 242 to 408 . Finally, Kohsaka and coworkers [34] performed fine epitope mapping and identified several autoantigenic La protein regions. Some of these regions were recognized by many sera, others by only a few. One of the commonly recognized regions included the aa 283-338. Interestingly, the epitope (aa 331-343) recognized by the anti-La mAb La4B6 is contained in all autoantigenic regions identified by the studies mentioned above. Moreover, it locates within a conserved functional region 
of the La protein. Finally, the mouse mAb La4B6 cross-reacts from human to mouse and thus represents an experimentally induced autoantibody.

\section{Acknowledgements}

This investigation was supported by a grant from the German Research Society (Deutsche Forschungsgemeinschaft, DFG (Ba 1145/1-2)). M. Bachmann is a recipient of a Hermann-and-Lilly-Schilling professorship granted by the 'Stifterverband für die Deutsche Wissenschaft'. I. Semsei is a recipient of a fellowship donated by the Alexander von Humboldt-Stiftung. M. Schwemmle is a recipient of an Isaac Lindenmann scholarship. The research of G. J. M. Pruijn has been made possible by a fellowship of the Royal Netherlands Academy of Arts and Sciences. The work of W. J. van Venrooij was supported in part by the Netherlands Foundation for Chemical Research (SON) with financial aid from the Netherlands Organization for Scientific Research (NWO).

\section{References}

1. Tan, E. M. 1989. Antinuclear antibodies: Diagnostic markers for autoimmune diseases and probes for cell biology. Adv. Inmunol. 44: 93-151

2. Van Venrooij, W. J., R. L. Slobbe, and G. J. M. Pruijn. 1993. Structure and function of La and Ro RNPs. Mol. Biol. Rep. 84: 113-121

3. Gottlieb, E. and J. A. Steitz. 1989. The RNA binding protein La influences both the accuracy and the efficiency of RNA polymerase III transcription in vivo. $E M B O \mathcal{f}$. 8: 841-850

4. Gottlieb, E. and J. A. Steitz. 1989. Function of the mammalian La protein: evidence for its activity in transcription termination by RNA polymerase III. EMBO f. 8: 851-861

5. Hendrick, J. P., S. Wolin, J. Rinke, M. Lerner, and J. A. Steitz. 1981. Ro small cytoplasmic ribonucleoproteins are a subclass of La ribonucleoproteins: Further characterization of the Ro and La small ribonucleoproteins from uninfected mammalian cells. Mol. Cell. Biol. 12: 1138-1149

6. Bachmann, M., K. Pfeifer, H. C. Schröder, and W. E. G. Müller. 1990. Characterization of the autoantigen $\mathrm{La}$ as a nucleic acid dependent ATPase/dATPase with melting properties. Cell 60: 85-93

7. Meerovitch, K., Y. V. Svitkin, H. S. Lee, F. Leibkowicz, D. Kenan, E. K. L. Chan, V. I. Agol, J. D. Keene, and N. J. Sonenberg. 1993. La autoantigen enhances and corrects aberrant translation of poliovirus RNA in reticulocyte lysate. $f$. Virol. 67: 3798-3807

8. Xiao, Q., T. V. Sharp, I. W. Jeffrey, M. C. James, G. J. M. Pruijn, W. J. van Venrooij, and $M$. J. Clemens. 1994. The La antigen inhibits the activation of the interferoninducible kinase PKR by sequestering and unwinding double-stranded DNA. Nucleic Acids Res. 22: 2512-2518

9. Topfer, F. T., T. Gordon, and J. McCluskey. 1993. Characterization of the mouse autoantigen La (SS-B): Identification of conserved RNA binding motifs, a putative ATP binding site and reactivity of recombinant protein with poly $(U)$ and human autoantibodies. F. Immunol. 10: 3091-3097

10. Pause, A. and N. J. Sonenberg. 1992. Mutational analysis of a DEAD box RNA helicase: the mammalian translation initiation factor eIF-4A. EMBO f. 11: 2643-2654

11. Semsei, I., H. Tröster, H. Bartsch, M. Schwemmle, G. Igloi, and M. Bachmann. 1993. Cloning of the autoantigen La/SS-B from a rat cDNA library: Detection of speciesspecific variations. Gene 126: $265-268$

12. Bachmann, M., W. J. Mayet, H. C. Schröder, K. Pfeifer, K.-H. Meyer zum Büschenfelde, and W. E. G. Müller. 1986. Association of $\mathrm{La}$ and Ro antigens with intracellular structures in HEp-2 carcinoma cells. Proc. Natl. Acad. Sci. USA 83: 7770-7774 
13. Bachmann, M., K. Pfeifer, H. C. Schröder, and W. E. G. Müller. 1987. The nucleocytoplasmic shuttling of the La antigen in CV-1 cells. Mol. Biol. Rep. 12: 239-240

14. Bachmann, M., Th. Zaubitzer, and W. E. G. Müller. 1992. The autoantigen La/SS-B: Detection and uptake by mitotic cells. Exp. Cell Res. 201: 387-398

15. Bachmann, M., H. Althoff, C. Selenka, D. Falke, and W. E. G. Müller. 1992. Translocation of the nuclear autoantigen La/SS-B to the cell surface of herpes simplex type 1 infected cells. Autoimmunity 12: 37-45

16. Laemmli, U. K. 1970. Cleavage of structural proteins during the assembly of the head of bacteriophage T4. Nature 227: 680-685

17. Matsudaira, P. 1987. Sequence from picomole quantities of proteins electroblotted onto polyvinylidene difuoride membranes. F. Biol. Chem. 262: 10035-10038

18. Holmes, D. S. and B. Quigley. 1981. A rapid boiling method for the preparation of bacterial plasmids. Anal. Biochem. 114: 193-197

19. Sambrook, J., E. F. Fritsch, and T. Maniatis. 1989. Molecular Cloning: A Laboratory Manual. Cold Spring Harbor Laboratory Press, Cold Spring Harbor, NY.

20. Henikoff, S. 1987. Unidirectional digestion with exonuclease III in DNA sequence analysis. Methods in Enzymology 115: 156-165

21. Slobbe, R. L., W. Pluk, W. J. van Venrooij, and G. J. M. Pruijn. 1992. Ro ribonucleoprotein assembly in vitro. $\mathcal{F}$. Mol. Biol. 227: 361-372

22. Studier, F. W. and B. A. Moffat. 1986. Use of bacterial phage T7 RNA polymerase to direct selective high-level expression of cloned genes. F. Mol. Biol. 189: 113-119

23. Bachmann, M., S. Chang, H. Slor, J. Kukulies, and W. E. G. Müller. 1990. Shuttling of the autoantigen $\mathrm{La}$ between nucleus and cell surface after UV irradiation of human keratinocytes. Exp. Cell Res. 191: 171-181

24. Chan, E. K. L., A.-M. Francoeur, and E. M. Tan. 1986. Epitopes, structural domains, and asymmetry of amino acid residues in SS-B/La nuclear protein. F. Immunol. 136: 3744-3749

25. Chan, E. K. L. and E. M. Tan. 1987. Human autoantibody-reactive epitopes of SS-B/La are highly conserved in comparison with epitopes recognized by murine monoclonal antibodies. F. Exp. Med. 166: 1627-1640

26. Smith, P. R., D. G. Williams, P. J. W. Venables, and R. N. Maini. 1985. Monoclonal antibodies to the Sjögren's syndrome associated antigen SS-B (La). F. immunol. Methods 77: $63-69$

27. Harley, J. B., M. O. Rosario, H. Yamagata, O. F. Fox, and E. Koren. 1985. Immunologic and structural studies of the Lupus/Sjögren's syndrome autoantigen, La/SS-B, with a monoclonal antibody. F. Clin. Invest. 76: 801-806

28. Pruijn, G. J. M. 1994. The La(SS-B) antigen. In W. J. van Venrooij and R. N. Maini, eds. Manual of Biological Markers of Disease. Kluwer Academic Publishers, Dordrecht. B4.2: $1-14$

29. Chan, E. K. L. and E. M. Tan. 1989. Epitopic targets for autoantibodies in systemic lupus erythematosus and Sjögren's syndrome. Curr. Opin. Rheumatol. 1: 376-381

30. Rauh, A. J. G., H. Hornig, and R. Lührmann. 1988. At least three distinct B cell epitopes reside in the C-terminal half of La protein, as determined by a recombinant DNA approach. Eur. F. Immunol. 18: 2049-2057

31. Sturgess, A. D., M. G. Peterson, L. J. McNeilage, S. Wittingham, and R. L. Coppel. 1988. Characteristics and epitope mapping of a cloned human autoantigen La. F. Immunol. 140: 3212-3218

32. Chambers, J. C., D. Kenan, B. J. Martin, and J. D. Keene. 1988. Genomic structure and amino acid sequence domains of the human La autoantigen. F. Biol. Chem. 263: 18043-18051

33. St Clair, E. W., D. S. Pisetsky, C. F. Reich, and J. D. Keene. 1988. Analysis of autoantibody binding to different regions of the human $\mathrm{La}$ antigen expressed in recombinant fusion proteins. F. Immunol. 141: 4173-4180

34. Kohsaka, H., K. Yamamoto, H. Fujii, H. Miura, and N. Miyasaka. 1990. Fine epitope mapping of the human SS-B/La protein. $\mathcal{F}$. Clin. Invest. 6: 2233-2244 